\title{
特別講演
}

\section{Hearing and Speech by Cleft Palate}

\author{
André Muller M. D.
}

\begin{abstract}
Adequate treatment of Cleft palate is of great importance for those who suffer from such malformations. Their relationship toward their environment is affected by several aspects : speech, audition, mimic disorders, aesthetical appearance.

The treatment is the duty of a multidisciplinary team working together in close collaboration.

Hearing impairment is very frequent by $\mathrm{CP}$ children due to tube dysfunction in relation to the velum malformation.

Considering the pathophysiological conditions, it is now generally recommended to correct every kind of anatomical malformation as soon as possible : early surgery allows the child to develop and acquire a normal phonological system at normal age. It permits an early functioning of velopharyngeal closure with better tube function and an early developement of the speech articulatory system.
\end{abstract}

Treatment of Cleft Palate is of great importance for those who suffer from such malformation. Their relationship toward their environment is affected by several aspects : phonation, audition, mimic disorders and aesthetical appearance.

Speech therapists and phoniatricians are primarily interested in their treatment, but this treatment is the task of a multidisciplinary team working together in close collaboration.

This malformation, which seems monstruous to the parents is originally a simple fissure without deformity, but which can be of various degrees of gravity, unilateral or bilateral. Osseous deformities are secondary and result from anomalies of the muscle equilibrium which depends principally on the function of the tongue. It is therefore important to obtain this equilibrium by early surgery which will improve speech and hypacousis by middle ear diseases.

The tongue, composed of 17 muscles, is of great muscular and nervous complexity. The mobility of the tongue is extraordinary and may play a role in the growth of environmental tissues. In newborns. the volume and the weight of the tongue is already half of that of an adult. Initially the most important motions of the tongue are the alimentary motions : they are the earliest and develop a maximum strength. Notice that at the 13 intrauterine week, the foetus is able to swallow and at the 5th intrauterine month he knows how to suck the thumb. For the newborn the suction requires the maximum strength.

It is a biologic general rule that the osseous growth is guided, eventually accelerated, by muscular equilibrium phenomenon. In the case of Cleft Palate the base of the tongue is spread at rest in the nasopharynx which is large and open is the front. The tip of the tongue, very mobile, goes from time to time in the nose pushing aside the palatal laminae with an upward displscement. Suction is impossible and the first buccal step of deglutition is totally impaired. The entire tongue is displaced upwards and backwards provoking a diverging action on the pterygoides process and modifying the growth of 
the palatal laminae and of the vomer.

Now to understand the causes of frequent hearing impairment by $\mathrm{CP}$ children we must go back to the pathophysiology of the velum. The movements of the velum are less complex and less powerful than those of the tongue, but they are precise and rapid.

The velum has 4 main muscles :

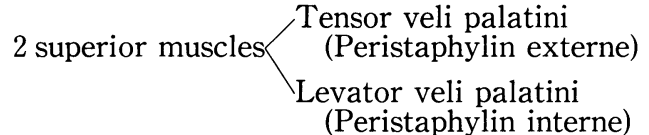

2 inferior muscles $\begin{aligned} & \text { pharyngostaphylin } \\ & \text { glosso staphylin }\end{aligned}$

Those muscles allow the velum
to raise
to stretch
to pull down by moving back
to pull down by pushing forward

This causes the isolation of the nasal cavity during swallowing and phonating and allows nasal breathing and several possibility of nasality.

In case of $\mathrm{CP}$, the function of these muscles is severely perturbated, causing an ET dysfunction and an insufficient velopharyngeal closure.

The three main functions of the ET are disturbed.

1) The protective function of the tube against reflux of naso-pharyngeal and food debris in the middle ear is compromised when the tube is abnormally large and also because the tube in newborns is shorter and more horizontal, which causes in any case problems in the supine position.

2 ) The clearance or drainage function by the mucociliary transport system of the ET with its pumplike action is perturbed.

3 ) The ventilatory function of the ET, which is always less efficient in normal children than in adults, is very poor is $\mathrm{CP}$ patients. The intermittent active opening by dilatation of the ET becomes rare : this creates high negative middle ear pressure.

A normal tube function depends mainly on the superior two muscles enumerated previously :

1) The Tensor Veli Palatini (Peristaphylin externe) opens the cartilaginous portion of the ET during swallowing, while simultaneously flattening the soft palate. One muscular bundle of this muscle is called the dilator tubae. Other bundles converge in a tendon which rounds the hamular process of the medial pterygoid laminae. This is specially dedicated to the swallowing function.

2 ) The Levator Veli palatini (Peristaphylin interne) which raises the soft palate during swallowing and only assists the tensor veli palatini in opening the ET. This muscle is mostly adapted to the phonation. LVP and TVP play an important role in the opening mechanism of the tube.

Reports of Ruding, Kriens. Dvoral, described in $\mathrm{CP}$ patients an inappropriate insertion of the levator veli palatini muscle which is inserted into the medial margin of the hard palate instead of inserting into the corresponding paired muscle in the medial raphe of the soft palate. The inappropriate insertions of these muscles causes atrophy and explains the inadequate function of sling for lifting the soft palate which is indispensable for good speech and for opening of the auditory tube.

Kaplan points out that side-to-side surgical closure of the velum without repositioning of the musculature, leaving the muscle fibers in a parallel position, rather than end-to-end positioning, does not give a normal sling for lifting the soft palate up and back.

Godbersen examining endoscopically newborns with $\mathrm{CP}$ noticed that the lateral part of the velum was closing the entrance of the tube creating middle ear diseases. He suggests that early veloplasty would ameliorate this tubal obstruction.

The tube dysfunction may be clearly seen in very young children first by otoscopy (with or without microscope) and by tympanometry which measures the eardrum elasticity. The eardrum vibration is the best when the air pressure is the same in the middle ear and in the ear canal. Tympanometry shows the difficulty of eardrum vibration, the impedance, and reverse the compliance, which results from difficulties of tube opening.

Early hearing impairment resulting from middle ear effusion before 2 years old has great importance on the development of verbal expression, especially since $\mathrm{CP}$ children are generally more subject to speech delay and difficulties in reading, this is also due to the velum and maxillary malformation and 
also to psycho affective relational troubles.

During the last 30 years, we encountered several contradictory opinions concerning the timing of Cleft Palate surgery. Maxillary surgeons prefer a late closure of the hard palate in order to preserve the maxillary and the face growth. Phoniaricians and logopedists recommend early palate closure, this to avoid the pressure mecanism and the bad positioning of the tongue, as mentioned before. This leads to a better speech development and less tube dysfunction with hearing impairment. If the child is operated on after two years, speech articulation disorders may not spontaneously disappear.

Actually in our CP centre, we follow the operational timing described by Psaume and Malek (Paris) at our 1986 IALP Congress in Tokyo, method which has been used by their authors since 1976 .

At the age of 6 weeks or earlier we make a mould and a plate of the palate which should be long enough to surpass the velum. This plate avoids the back positioning of the tongue and favours a better position of the hard palate and of the vomer. This plate is maintained in place continuously until the veloplasty and even 3-4 weeks after this operation.

Veloplasty is performed at 3 months this operation is carried out taking great care not to damage the fibromucosal tissue of the palate.

Closing of the lip and the palate (in unilateral cleft) occurs at 6 months and a second plate is made which protects the suture and diminishes the risk of a small nose-palate connection.

In case of bilateral cleft this palate closure is done in two steps, at 6 and 8 months. Altogether this early operational timing gives better anatomical conditions. Occlusive and constrictive consonants may appear at the normal age and the tube function is improved. The early veloplasty normalizes the problems of pressure in the nasopharynx which is essential to the tube function, producing a better tube opening and less middle ear infections.

Speech, which reflects the personality, depends on :
hearing
nasal respiration
lips
jaw-teeth

velar closure

Concerning speech, during many years the age of 18 months was considered the best for Cleft interventions, than it had been proved that if this intervention happened after 2 years, it was very difficult to get rid of bad compensatory articulation habits, like glottal stops. Actually, 2 years is no longer considered as the beginning of language. One admits that the child is competent for communication since birth and capable to interact. Some physiologists are of the opinion that, if neurons are not used early, they disappear definitely.

But it is especially when he starts to articulate, pronouncing phonems, that the $\mathrm{CP}$ child will get into trouble. Psychologic factors, like those caused by parents rejecting more or less their child who is not intelligible and who has possibly hearing impairment, may diminish the quality of the feedback, which is so important for the language structure and development.

After the end of the first year the child is in possession of the principles of language organization.

As it has been observed, all 6 weeks children give glottal stops, but at about 8 weeks they start exercising the velum, giving vibrations between the back part of the tongue and the velum. Those vibrations are replaced at 3 months by simple posterior articulatory movements. This is not possible for a $\mathrm{CP}$ child as the velum is not competent, so that the glottal stops remain. Therefore we need to close the velum as early as possible this means at 3 months and to close the hard palate and the lip at 6 months.

With this early chronology, functional anatomical conditions are established on time. This permits :

1 ) early functioning of velopharyngeal closure avoiding nasopharyngeal enlargement.

2 ) early development of the articulatory system.

3 ) early starting of speech.

This early chronology avoids compensation or articulatory substitutions, which are always very difficult for the speech therapist to eliminate.

If at 20 months a CP child has not been operated and is not in condition to be understood because to much nasality or very poor intelligibility with glot- 
tal stop subsitutions, he will give up talking or take the habit of shouting or just making intonations or mimics. However, the early chronology allows the child to develop and acquire a normal phonological system at the normal age.

Early surgery must be absolutely completed by providing information to the parents : parents should be able to complement the anatomical and functional profit obtained by this surgery.

In $\mathrm{CP}$ children with persistent rhinophonia or hypernasality it is essential to appreciate the velopharyngeal mechanism as these exists a great anatomical and functional variability of this closure.

What may contribute to the persistence of hypernasality?

1) Anatomical aspects : structural deviations of the nasal cavities influence of malocclusion and orthodontic

2) Functional aspects :

disordered articulatory behavior disordered oral behavior environmental problems

\section{Speech resonance or hypernasality}

have three origin : nasal

$$
\text { oral }
$$

a ) nasal resonance : easy ot control by rhinoscopy (deviated septum, hypertrophic turbinates?)

b) oral resonance : nasality caused by hypertrophic tonsils with breathing, swallowing and articulatory difficulties and provoking blockage of the velum and of lateral pharyngeal movenents.

c) nasopharyngeal

resonance : this is the VPI or Velopharyngeal Insufficiency.

VPI has been well described in a main report at our Tokyo Meeting 1986 by Hirschberg.

VPI may be of congenital or acquired origin, may be organic or functional, and very often we have to deal with congenital short palate or also with submucous cleft and secondarily with paretic syndroms from neurological diseases.

The primary speech defests of VPI are hypernasality and nasal escape. Hypernasality is a change in voice quality resulting from the acoustic coupling of naso and oropharynx through the open or incompetent VP sphincter (evident only by vowels) .

It exists several tests and methods for evaluation of velopharyngeal insufficiency. Simple functional tests like Czermak's mirror test, Gutzmann's A-test, phonendoscope test give quick and suitable information on nasality but nasopharyngeal fibroscope is the best method to test velopharyngeal closure during speech and this in every day practice of a cleft palate clinic. With this $2 \mathrm{~mm}$ flexible fibroscope the velopharyngeal view is monitored on a TV screen and is recorded as a routine clinical examination. Analysis of the valving pattern is done. It gives also the possibility to visualize the nasal surface of the soft palate, the Eustachian tube orifices, the size and shape of adenoid tissue and tonsillar tissue, the epiglottis and laryngeal structures. During closure of the velopharyngeal valve of the circular type, the Passavant's ridge - when it exists - can be covered by the lateral velopharyngeal walls and the velum as well. In this case some authors recommended the forced sucking manoeuvre which provokes the appearance of the shape and level of the Passavan's ridge : during sucking the velum descends and the dorsum of the tongue is raised. Thus, the velopharyngeal valve remains open permitting observation of the lateral walls movements, from which the nasal resonance is also dependent.

This fibroscopy provides reliable indications for the type of the secondary surgery for improving VP closure, pharyngeal flap operation in particular.

In case of persistance of severe hypernasality, it is the role of the $\mathrm{CP}$ team, judging several factors and circumstances (anatomy, compensatory mechanisms, intelligence, child and family collaboration) to determine whether a better result can be obtained by surgery or by speech therapy, or by both, which are complementary. The team has then to decide the necessity of a pharyngoplasty which is in many cases the only way to improve speech and at the same time hearing. In our statistics $9 \%$ of our CP 
children needs a pharyngoplasty, operation which is usually performed between 4 and 6 years, also in case of congenital short palate and submucous cleft palate. We prefer the superiorly based flap which seems to establish better physiological conditions and permit the closure of a larger gap. Pharyngoplasty is always preceded by adenotomy (adenoids trouble the dynamic function of the ET during swallowing and may cause serous otitis), and eventually tonsillectomy in case of enlarged tonsils.

The favorable effect on hearing is probably due to better separation of the oro and nasopharynx, as well as by the fact that the pharyngeal flap improves the position and function of the Levator Veli Palatini, reducing the reflux of pharyngeal contents into the orifice of the Tube, facilitating also its opening.

Speech conditions are usually very much improved by a pharyngoplasty, but speech therapy is always needed for a few months after the operation.

Characteristics of CP Speech are well known, among them.glottal stops and compensatory articulation errors. For evaluation of hypernasality or rhinophonia most authors use a 5 or 7 points descriptive scale. Speech intelligibility is disrupted fare more by the misarticulation of consonants than it is by the nasalisation of vowels. Following Isshiki, they are now a few simple intelligibility tests which gives an idea about the quality of speech as a whole, showing to which extent the child is understood by its surroundings.

Early surgery of $\mathrm{CP}$ allows early and better results of speech therapy : this should start by providing information to the parents, insisting on their role of speech educator, stimulating the vocal and articulation production, this with a good feedback in play and pleasure. We must show them how to practice blowing exercises and mouth and face motions after the age of 10-11 months. If the child has difficulties to speak, speech education should start between 18 and 24 months in presence of the parents. But a regular speech education will then follow at 3 years, sometimes in a group therapy. It is essential to eliminate glottal stops and compensatory articulation and increase velopharyngeal contacts. To be effective this treatment of hypernasality must be frequent and intensive, like a drill, using some times the technique of the speech bulb.

In conclusion it seems to be wise to correct every kind of anatomical malformation as soon as possible, provided we have the technical possibilities. The CP team will obtain in this way an earlier and better functioning of the velum which will result in better tube conditions with less possibilities of hearing impairment and in an earlier beginning of speech and language. 